\title{
Alternative alla detenzione: quali prospettive in Europa? Analisi, buone prassi e ricerca in sette Paesi dell'Unione Europea
}

\author{
Giorgia Stefani
}

\begin{abstract}
Riassunto
L'articolo propone alcune riflessioni su quanto emerso dall'attività di ricerca nell'ambito del progetto "Reducing prison population: advanced tools of justice in Europe", finalizzato al miglioramento delle conoscenze e allo scambio di buone pratiche in tema di misure cautelari e alternative alla detenzione in sette paesi europei (Italia, Bulgaria, Lettonia, Francia, Germania, Regno Unito (Scozia), Romania).
\end{abstract}

\section{Résumé}

L'article propose des réflexions sur les résultats de l'activité de recherche menée dans le cadre du projet «Reducing prison population : advanced tools of justice in Europe » («Réduction de la population carcérale : les avancées des instruments de justice en Europe »). Ce projet a pour but l'amélioration des connaissances et l'échange de bonnes pratiques dans le domaine des mesures conservatoires et alternatives à la détention dans 7 pays européens (Italie, Bulgarie, Lettonie, France, Allemagne, Royaume-Uni - Écosse -, Roumanie).

\section{Abstract}

This article proposes some reflections on the results of the research conducted within the framework of the project "Reducing prison population: advanced tools of justice in Europe". The aim of this project is to improve knowledge and exchange of best practice in the domain of protective orders and alternatives to imprisonment in seven European countries (Italy, Bulgaria, Latvia, France, Germany, United Kingdom (Scotland) and Romania).

Key words: reducing prison population; alternatives to imprisonment; precautionary measures; tools of justice; Europe.

\section{Introduzione.}

Il progetto "Reducing prison population: advanced tools of justice in Europe", finanziato dal Programma europeo "Criminal Justice", è volto al miglioramento delle conoscenze e allo scambio di buone pratiche in tema di misure cautelari e alternative alla detenzione. Coordinato dall'Associazione Comunità Papa Giovanni XXIII vede coinvolti in attività di ricerca il C.I.R.Vi.S-SDE (Centro Interdisciplinare di Ricerca sulla Vittimologia e sulla Sicurezza Dipartimento di Sociologia e Diritto dell'Economia) dell'Università di Bologna, Synergia, società milanese di ricerca sociale, consulenza organizzativa e formazione, la Società Internazionale di Criminologia (Francia), l'Università di Dundee (Scozia), l'Università di Scienze applicate per la pubblica amministrazione di Brema (Germania), il Centro per le politiche pubbliche Providus (Lettonia), le organizzazioni non governative IGA (Bulgaria) e Generatie Tanara (Romania).

Il diritto penale ed il diritto penitenziario in ciascuno Stato, per lungo tempo considerati oggetto di esclusivo interesse nazionale, sono sempre più influenzati dalle istituzioni europee;

\footnotetext{
- Associazione Comunità Papa Giovanni XXIII, Rimini.
} 
in questa prospettiva l'idea progettuale è nata e si è sviluppata sulla base di tre considerazioni:

(a) le carceri europee, e in particolare quelle italiane, sono sovraffollate: questo viola $i$ diritti fondamentali dei detenuti ed è causa di numerosi problemi anche per il personale penitenziario;

(b) le attuali politiche sociali offrono poche possibilità per il reinserimento sociale di exdetenuti;

(c) le politiche penitenziarie prestano poca attenzione ai bisogni e ai diritti delle vittime.

Attraverso attività di ricerca sul campo e lo scambio di buone pratiche in materia di alternative al carcere il progetto intende affrontare tutte e tre le questioni.

Il tema del superamento del regime penitenziario è di grande attualità: una recente relazione del Comitato per gli affari giuridici del Consiglio d'Europa ha identificato nel sovraffollamento il problema principale delle carceri europee e la necessità di un progressivo ampliamento delle misure alternative alla detenzione è da lungo tempo richiamata da più parti come imprescindibile impegno.

Già nel 1990, infatti, l'assemblea generale delle Nazioni Unite ha adottato le Regole di Tokyo (Tokyo Rules) con l'obiettivo di incoraggiare l'utilizzo delle misure non carcerarie sottolineando l'importanza della partecipazione pubblica nello sviluppo di queste ultime. Anche il Consiglio d'Europa, dopo un'iniziale attenzione rivolta al sistema detentivo, attraverso una serie di Raccomandazioni R(92)16, $\mathrm{R}(2000) 22$ e $\mathrm{R}$ (2010)1 ha concentrato la sua attività sulle misure alternative alla detenzione, definendole, più propriamente "community sanctions". La Commissione Europea, tramite il
Libro verde sulla detenzione $^{1}$, nel 2011 ha voluto valutare in che misura la custodia cautelare e le condizioni carcerarie danneggiassero la fiducia reciproca e l'effettiva cooperazione giudiziaria tra gli Stati membri e, in senso più ampio, il ruolo dell'Unione in quest'ambito. Dall'esame dei numerosi contributi ricevuti in risposta al Libro verde dagli Stati membri e dalle organizzazioni della società civile è emersa, soprattutto, l'importanza di garantire un'attuazione adeguata e tempestiva dell'attuale normativa dell'UE volta a promuovere soluzioni alternative alla detenzione.

Il sovraffollamento carcerario, infatti, impedisce non solo l'attuazione di programmi trattamentali, ma anche il rispetto dei più elementari diritti dei detenuti. Il problema riguarda molti sistemi penali: secondo il recente rapporto SPACE I 2013 del Consiglio d'Europa ${ }^{2}$, pubblicato a febbraio 2015, che fotografa la situazione al 2013 dei 49 paesi della più antica istituzione europea, il sovraffollamento riguarda 21 dei 49 penitenziari dei paesi presi in esame.

I vari Stati membri hanno sperimentato differenti rimedi al problema: lo scambio di buone prassi che il progetto permette è una risorsa fondamentale per la soluzione degli specifici problemi di ciascun sistema penitenziario nazionale.

\section{Lo stato dell'arte e le tendenze attuali.}

\footnotetext{
1 Commissione Europea, Libro Verde. Rafforzare a fiducia reciproca nello spazio giudiziario europeo - Libro verde sull'applicazione della normativa dell'UE sulla giustizia penale nel settore della detenzione, 2011, consultabile al sito:

http://www.europarl.europa.eu/meetdocs/2009 201 4/documents/com/com com

(2011)0327_/com_com(2011)0327_it.pdf

2 Aebi M.F., Delgrande N., SPACE I - Council of Europe Annual Penal Statistics: Prisons populations, Survey 2013, Consiglio d'Europa, Strasburgo, 2015.
} 
Quando si parla di misure non carcerarie a livello europeo il Comitato dei Ministri del Consiglio d'Europa per mezzo della Raccomandazione (92)16, rifacendosi al termine anglosassone community sanctions, identifica le misure che mantengono il condannato nella comunità ed implicano una certa restrizione della sua libertà attraverso l'imposizione di condizioni e/o obblighi e che sono eseguite dagli organi previsti dalle norme in vigore.

L'attività di ricerca sinora condotta ha permesso di compiere un'analisi del quadro europeo in tema di misure non carcerarie e una ricognizione delle caratteristiche e dell'avanzamento delle alternative alla detenzione, sia in fase pre che post processuale, in ogni paese coinvolto nel progetto.

Il rapporto sul quadro politico delle alternative alla detenzione entro il contesto europeo, condotto nell'ambito del progetto dall'Istituto di Criminologia di Leuven per conto della Società Internazionale di Criminologia, ha ampiamente evidenziato come non sia solamente il crimine in sé a determinare l'entità della popolazione carceraria ma come, al contrario, questa dipenda da numerosi fattori correlati ${ }^{3}$, identificati da alcune ricerche ${ }^{4}$ in tre categorie:

(1) meccanismi interni, cioè fattori connessi al sistema di giustizia penale, come, ad esempio, la

\footnotetext{
${ }^{3}$ De Vos H., Gilbert E., Aertsen I., Reducing prison pupolation overview of the legal and policy framework on alternatives to imprisonment at the European level, 2014, consultabile al sito: http://www.reducingprison.eu/downloads/files/Red ucingprisonpopulationEuropeanframework_FIN_101 014.pdf

4 Beyens K., Snacken S. Eliaerts C., Barstende muren, overbevolkte gevangenissen: omvang, oorzaken en mogelijke oplossingen, Kluwer, Bruxelles, 1993, citato da De Vos H., Gilbert E., Aertsen I, op. cit..
}

legislazione, la polizia, l'azione penale, la custodia cautelare, le condanne;

(2) fattori di disturbo, tra cui, il ruolo dei media, l'opinione pubblica e il clima politico;

(3) fattori esterni, connessi ad evoluzioni demografiche ed economiche.

Attraverso lo studio della letteratura nazionale in ogni paese coinvolto, raccolto in sette rapporti specifici, è stato possibile ottenere una fotografia della situazione carceraria nei diversi Paesi, sia dal punto di vista socio-demografico che da quello delle politiche sociali.

Per quanto riguarda la composizione sociodemografica della popolazione detenuta, i paragoni tra Paesi devono essere compiuti con molta cautela poiché l'entità della popolazione carceraria riflette una molteplicità di fattori, come, ad esempio: il livello di criminalità, sistemi giuridici e ordinamenti giudiziari penali differenti, la lunghezza e la modalità di applicazione della custodia cautelare, la diversità nell'utilizzo di misure alternative alla detenzione nei vari gradi di giudizio. L'eterogeneità delle informazioni disponibili nei singoli rapporti nazionali rende difficile una comparazione dei dati raccolti, per tale motivo, in questo articolo si è scelto di fare riferimento alle informazioni riportate nell'ultima indagine "SPACE I" condotta, per il Consiglio d'Europa, dall'Istituto di Diritto Penale e Criminologia dell'Università di Losanna; che contiene informazioni sulle Amministrazioni Penitenziarie presenti nei 49 Stati membri del Consiglio d'Europa, e che dal 2004 garantisce la raccolta, l'analisi e 
l'interpretazione dei dati tramite una metodologia comune 5 .

Un dato importante riportato nell'indagine è quello che fa riferimento al tasso di detenzione, ossia il numero di detenuti ogni 100.000 abitanti: il tasso medio europeo è di 134 detenuti su 100.000 abitanti, in leggero aumento rispetto a quello del 2012 (che era di 127 detenuti su 100.000 abitanti).

Il problema del sovraffollamento dei penitenziari interessa prevalentemente l'Italia: con un tasso di sovraffollamento pari al 148\%, in aumento rispetto al $2012(+3 \%)$, anche se, tra i paesi partecipanti al progetto, tassi elevati si registrano anche in Francia (117\%) e Romania (116\%).

Dall'analisi dei vari rapporti, emerge chiaramente come la proporzione di donne in carcere in tutti Paesi coinvolti abbia valori molto contenuti e omogenei: gli scarsi numeri di detenute in tutta Europa portano a trascurare questa categoria nella predisposizione di politiche specifiche. La Risoluzione del Parlamento europeo del 13 Marzo 2008 ha, invece, evidenziato come la condizione sociale delle donne detenute sia caratterizzata da numerosi svantaggi e come sia necessario "tenere maggiormente presenti le specificità femminili e il passato spesso traumatico delle donne detenute" e ha raccomandato agli Stati Membri "che pene sostitutive della detenzione siano comminate in misura maggiore, come le alternative sociali, in modo particolare per le madri"'.

Altri elementi interessanti deducibili dal rapporto SPACE I 2013 riguardano il rapporto tra

\footnotetext{
${ }^{5}$ Aebi M.F., Delgrande N., SPACE I, op.cit.

${ }^{6}$ Risoluzione del Parlamento europeo del 13 marzo 2008 sulla particolare situazione delle donne detenute e l'impatto dell'incarcerazione dei genitori sulla vita sociale e familiare, consultabile al sito: http:/ /www.europarl.europa.eu/sides/getDoc.do?pu bRef $=-/ /$ EP $/ /$ TEXT+TA+P6-TA-2008-

0102+0+DOC+XML+V0//IT
}

numero di detenuti e personale preposto: in Italia il rapporto è di 1,7 detenuti ogni agente penitenziario; il dato è decisamente inferiore a quello di altri Paesi del partenariato, quali, ad esempio, la Romania e la Lettonia, che presentano un rapporto tra detenuti e staff rispettivamente di 7 a 1 e 3 a 1.

Come sostenuto da Gresham Sykes, sociologo americano, le privazioni e le frustrazioni della prigione moderna "possono essere tanto dolorose quanto $i$ maltrattamenti fisici che hanno sostituito": il $17 \%$ delle morti in carcere in Europa, infatti, avviene per suicidio, che è la seconda causa di morte dopo quella naturale. In Francia e Germania, nel 2012 la percentuale di detenuti morti per suicidio è stata pari al 57,8 e al 47,9\%

La presenza di stranieri tra i detenuti in Europa è di circa il 15\%, ma dal Rapporto SPACE I si evince una forte disomogeneità tra $\mathrm{i}$ dati forniti dai diversi Paesi coinvolti dall'indagine. Tra i paesi interessati dal progetto i valori più bassi si registrano tra quelli dell'est europeo (Bulgaria, Romania e Lettonia), tradizionalmente paesi di emigrazione e non di immigrazione. Italia, Francia e Germania hanno, invece, il più alto numero di detenuti stranieri.

La questione è sicuramente una delle emergenze da affrontare nel sistema carcerario italiano dove l'alta percentuale di stranieri è giustificata dal fatto che nel paese per un immigrato irregolare è difficile trovare misure cautelari alternative al carcere: gli stranieri senza permesso di soggiorno, infatti, non avendo un domicilio stabile, non possono essere tenuti agli arresti domiciliari ${ }^{7}$. La conseguenza di questo processo ¿̀ l'aumento dell'esclusione sociale, che si ripercuote negativamente sulla società sia in termini di costi economici (mantenimento in

\footnotetext{
${ }^{7}$ Gonnella P., Detenuti stranieri in Italia. Norme, numeri e
} diritti, Editoriale scientifica, Napoli, 2015. 
carcere) che sociali (difficile integrazione, alto rischio di recidiva, maggiore insicurezza sociale, ecc.).

E' opinione condivisa dal partenariato, comunque, che $\mathrm{i}$ detenuti stranieri siano considerati svantaggiati rispetto agli autoctoni: sebbene dal punto di vista giuridico i diritti siano i medesimi, la letteratura francese e quella tedesca hanno mostrato come quest'apparente uguaglianza di fatto nasconda una disparità effettiva, in particolare per quanto riguarda l'accesso ai diritti, l'istruzione a lungo termine, la liberazione anticipata. Per cercare di limitare questi problemi, la decisione quadro 2008/219/GAI, relativa all'applicazione del principio del reciproco riconoscimento alle sentenze penali che infliggono pene detentive o misure privative della libertà personale, ai fini della loro esecuzione nell'Unione europea, consente il trasferimento dei detenuti condannati nello Stato membro della loro nazionalità, residenza abituale o ad un altro Stato membro con cui abbiano stretti legami. In alcuni casi, il trasferimento della persona condannata può aver luogo anche senza il consenso di questa persona, tuttavia, occorre ricordare che, giacché lo scopo della decisione è quello di sostenere il reinserimento sociale della persona condannata, il trasferimento allo Stato di nazionalità della persona non è sempre compatibile con questo obiettivo, in particolare in mancanza di vincoli familiari o professionali.

Sempre secondo l'indagine SPACE I il costo medio di gestione di un detenuto in carcere è di 97 Euro al giorno, ma ci sono significative disparità tra i vari paesi e, normalmente, il costo di mantenimento è inversamente proporzionale al tasso di detenzione del Paese. Nei Paesi coinvolti il costo medio di mantenimento giornaliero va dai 14,72 Euro della Lettonia ai 128 dell'Italia.

Tra i detenuti si segnalano tassi di consumo di stupefacenti complessivamente più alti di quelli riscontrati tra la popolazione generale e modelli di consumo più dannosi; studi recenti indicano, infatti, che una percentuale di detenuti compresa tra il 5\% e il $31 \%$ ha assunto stupefacenti per via parenterale almeno in un'occasione ${ }^{8}$. Tutti gli Stati Membri garantiscono servizi di assistenza ai tossicodipendenti in carcere, sebbene $\mathrm{i}$ tipi di servizi e la loro disponibilità vari notevolmente da paese a paese. La maggior parte dei paesi ha istituito partenariati tra $\mathrm{i}$ servizi sanitari carcerari e gli erogatori dei medesimi servizi a livello territoriale, per garantire l'educazione sanitaria e il trattamento all'interno del carcere, e per assicurare la continuità dell'assistenza dall'inizio della detenzione alla scarcerazione.

\section{Le misure alternative alla detenzione.}

L'analisi della letteratura e le interviste a testimoni privilegiati hanno permesso di compiere un approfondimento circa le attuali alternative al carcere (pre e post sentenza) e hanno messo in luce che il dibattito sulle misure non carcerarie sembra interessare, in maniera più o meno diretta, tutti i Paesi coinvolti nel progetto, testimoniando un significativo incremento, in tali realtà, della necessità di sviluppare strategie in materia di esecuzione penale esterna e probation.

Il probation secondo la definizione del Comitato

\footnotetext{
${ }^{8}$ Osservatorio Europeo Droghe e Tossicodipendenze (OEDT), Relazione europea sulla droga. Tendenze e sviluppi, 2014, consultabile al sito:
} 
dei Ministri del Consiglio d'Europa per mezzo delle Raccomandazioni n. R(2010)1 e n. R(92)16 descrive l'esecuzione in area penale esterna di sanzioni e misure, definite dalla legge ed imposte ad un autore di reato. Comprende una serie di attività ed interventi, tra cui il controllo, il consiglio e l'assistenza, mirati al reinserimento sociale dell'autore di reato e volti a contribuire alla sicurezza pubblica9.

Caratteristica fondamentale del probation è l'imposizione di obblighi comportamentali nei confronti dei condannati: il giudice, infatti, contestualmente all'applicazione della misura, fissa delle prescrizioni che il soggetto deve rispettare. Da qui il duplice compito dell'agente di probation che, da una parte, deve controllare l'osservanza dei precetti imposti riferendo all'autorità giudiziaria le eventuali violazioni, dall'altra deve affiancare chi sta scontando la misura con un ruolo di sostegno.

Le persone che sono in una sanzione o misura alternativa al carcere sono generalmente sotto il controllo di agenzie di probation di ogni paese.

Il rapporto sul quadro politico delle alternative alla detenzione in ambito europeo, condotto dall'Istituto di Criminologia di Leuven per conto della Società Internazionale di Criminologia ${ }^{10}$, ha mostrato come le alternative alla detenzione possano essere utilizzate nell'ottica di una strategia cosiddetta "front-door", cioè per evitare l'ingresso in carcere, oppure come strumento per favorire il reinserimento sociale del detenuto, permettendo l'uscita anticipata dal penitenziario

http://www.emcdda.europa.eu/attachements.cfm/att _228272_IT_TDAT14001ITN.pdf

${ }^{9}$ Ministero della Giustizia, Probation, consultabile al sito:

http://www.giustizia.it/giustizia/it/mg_2_3_1.wp

${ }^{10}$ De Vos H., Gilbert E., Aertsen I., op.cit. in sostituzione del periodo finale di una pena detentiva ("back-door"). Queste modalità rispondono a due diverse strategie di politica criminale: nel primo caso, con la concessione di misure alternative dallo stato di libertà si evita l'esperienza carceraria, con tutto quello che comporta, ai condannati ad una pena mediobreve; nel secondo caso, invece, si permette, in una logica di trattamento progressiva, che l'ultima parte di pene medio-lunghe sia espiata (totalmente o parzialmente) sul territorio.

Le misure alternative alla detenzione possono essere applicate prima e dopo una sentenza di condanna definitiva: prima del processo il ricorso eccessivo alla custodia cautelare e la durata di tale misura, infatti, è una delle cause principali del sovraffollamento carcerario.

\subsection{Le misure applicabili in fase cautelare.}

Il Consiglio d'Europa, nell'ambito del rapporto SPACE II-2013 relativo alle misure non custodiali ${ }^{11}$, raggruppa le alternative alla detenzione applicabili prima di una sentenza di condanna in:

- Alternative alla custodia cautelare con la supervisione di agenzie di probation, ad esempio tramite controllo elettronico, che consente di localizzare la persona mediante l'impiego di diverse tecniche, o con gli arresti domiciliari, in cui è richiesto alla persona di rimanere in modo permanente presso il proprio domicilio di residenza.

- Sospensione condizionale del processo, durante la quale, prima di un qualsiasi accertamento di colpevolezza, l'autorità giudiziaria

11 Aebi M.F., Chopin J., SPACE II- Council of Europe Annual Penal Statistics: Persons Serving non-custodial 
competente dispone la sospensione del procedimento per un tempo determinato, al fine di valutare il comportamento dell'imputato in quel periodo o per consentire la mediazione o la procedura di conciliazione.

- Rinvio nella pronuncia della sentenza, quando la decisione sulla pena da infliggere è rinviata e il condannato sottoposto ad un periodo di probation al termine del quale la persona può essere condannata o il procedimento può essere archiviato.

- Attività di mediazione vittima-reo la mediazione è una modalità per risolvere i conflitti tra il reo e la vittima di reato. Non è una misura alternativa vera e propria, ma di essa a volte può occuparsi l'ufficio di probation.

- Altre.

Le misure sono, tuttavia, molteplici e si caratterizzano per l'estrema peculiarità delle singole situazioni.

La legislazione nazionale di alcuni Paesi partecipanti al progetto (Scozia, Bulgaria, Romania, Lettonia), ad esempio, prevede che l'autorità giudiziaria possa concedere, quale sanzione alternativa alla carcerazione preventiva, la libertà dietro al pagamento di cauzione, eventualmente subordinata al rispetto di alcune condizioni. Questo strumento contribuisce, indubbiamente, alla riduzione del numero dei detenuti in attesa di giudizio che rappresentano una parte considerevole della popolazione carceraria europea, ma le voci critiche di alcuni esperti intervistati nell'ambito del progetto fanno riferimento a come il ricorso alla cauzione sia

sanctions and measures in 2013. Survey 2013, Consiglio riservato solamente ai soggetti facoltosi, contribuendo ad aumentare il divario tra soggetti abbienti e meno abbienti.

La misura degli arresti domiciliari, che permette all'imputato di scontare la pena presso la sua abitazione senza però allontanarsi da essa è ampiamente utilizzata (Italia, Lettonia, Romania, Bulgaria), anche se spesso criticata perché ritenuta priva di una finalità riabilitativa.

Novità della normativa italiana è l'utilizzo anche in fase cautelare dell'istituto della messa alla prova (probation), istituito nel 2014 ma già utilizzato nel procedimento penale minorile, che, oltre ad agevolare il reinserimento sociale del reo, in un'ottica di giustizia riparativa permette allo stesso di riparare il reato commesso, attraverso il lavoro gratuito alla comunità, fornendo così una prova concreta del riconoscimento del suo errore.

\subsection{Le misure applicabili dopo una sentenza di condanna.}

Dopo una sentenza di condanna definitiva le alternative alla detenzione sono più numerose. Il rapporto SPACE II ${ }^{12}$ le classifica in:

- Sospensione totale della pena con messa alla prova. in questo caso il giudice può stabilire le condizioni per la sospensione della pena in un certo periodo. L'esecuzione della sanzione è sospesa, ma la persona è obbligata ad attenersi a determinate condizioni.

- Sospensione parziale della pena con messa alla prova: in questo caso il giudice pronuncia una sentenza di condanna alla reclusione, di cui una parte è scontata sotto custodia (anche

d'Europa, Strasburgo, 2015.

12 Ibidem. 
semi-libertà o libertà vigilata) e una parte è sospesa.

- Perdono condizionato o semilibertà con messa alla prova, concesso a seguito del rispetto delle prescrizioni allegate alla sentenza (ad es. il risarcimento dei danni alla vittima, la terapia di disintossicazione da alcol o droga...).

- Servizio alla comunità, cioè lavoro non retribuito di pubblica utilità che può essere disposto come una sanzione a sé stante, come una condizione per la sospensione condizionale o la liberazione condizionale o come una sanzione supplementare.

- Monitoraggio elettronico.

- Detenzione domiciliare.

- Semilibertà, in questo caso il reo deve trascorrere un certo periodo di tempo (le notti, il fine settimana o alcuni giorni della settimana) in prigione e un certo periodo di tempo fuori dal carcere.

- Richiesta di trattamento, che può essere pronunciata in diversi momenti del procedimento penale, in caso di soggetti tossicodipendenti e alcoldipendenti, o per soggetti con disturbi mentali o condannati per reati sessuali.

- Liberazione condizionale con messa alla prova in questo caso viene disposta la liberazione condizionale del detenuto prima del termine di fine pena con individuali o specifiche condizioni.

- Ordini misti, che combinano due o più delle suddette misure

- Altre.

La classificazione, naturalmente, non è esaustiva e ogni sistema nazionale presenta caratteristiche peculiari. E' opinione condivisa dal partenariato che le misure di probation occupino un posto di privilegio nelle misure alternative alla detenzione. Storicamente questa misura è stata la prima veramente alternativa alle pene privative della libertà: la sua finalità di riabilitazione del condannato costituisce un elemento particolarmente rilevante al fine del reinserimento nella società. Attraverso questo istituto, infatti, l'oggetto del processo si sposta dal reato alla persona offrendo alla stessa la possibilità di adoperarsi e dare una svolta alla propria vita. Molti sono i paesi del partenariato che possono vantare un buon livello di sviluppo dei propri servizi di probation, anche se esistono divergenze in riferimento al livello di qualità all'applicazione concreta e alla varietà delle prestazioni offerte (mediazione penale, supporto alle vittime ecc.). I termini in cui fanno riferimento agli utenti del servizio (ad esempio, delinquenti o clienti o utenti del servizio) sono diversi e talvolta indicativi del modo in cui la probation comprende e sceglie di rappresentare il suo lavoro ${ }^{13}$.

Anche il lavoro di pubblica utilità è valutato positivamente dagli esperti intervistati: principio della misura è che il soggetto, lasciato in libertà, svolga a titolo di pena un certo numero di ore di lavoro, gratuitamente, in un organismo o a vantaggio di determinate persone; l'attività è svolta in termini riparativi per la comunità. La peculiarità sta nel fatto che la società partecipa in modo attivo all'esecuzione della pena ed alla riabilitazione del reo, attraverso degli organismi

\footnotetext{
${ }^{13}$ Herzog Evans M., "What's in a name: Penological and institutional connotations of probation officers' labelling in Europe", in EuroVista n. 2, 2013, pp. 121-133.
} 
presso i quali deve essere svolto il lavoro.

Nel dibattito relativo ai dispositivi di controllo elettronico, utilizzati attraverso modalità diverse in Francia, Germania, Scozia e Italia, si trovano opinioni favorevoli e altre che sono contrarie, sia nell'opinione pubblica, sia tra le organizzazioni professionali del settore penale ${ }^{14}$. Nell'appoggiare l'utilizzo di tale misura si fa riferimento alla necessità di ottenere la certezza della pena, alla parziale soluzione del sovraffollamento e al tentativo di rendere più sicura l'esecuzione di una misura alternativa alla detenzione. In Scozia, un esperto intervistato nell'ambito del progetto ha fatto presente l'esistenza di un divario tra la legislazione e la prassi in materia di sorveglianza elettronica: la tecnologia, infatti, non raggiunge il livello di sofisticazione tale per attuare il controllo al livello desiderato. ${ }^{15}$ In Italia, il braccialetto elettronico, introdotto nel 2001, non ha mai riscosso successo e molte sono state le polemiche sul suo utilizzo e, soprattutto, sui costi. Nell'ottobre 2012 la Corte dei Conti ha evidenziato "una notevole sproporzione tra gli elevati costi e il numero veramente esiguo" dei dispositivi utilizzati e ha dichiarato la gestione della misura "antieconomica e inefficace"16. Un esperto italiano,

14 Leonardi F., "La sorveglianza elettronica come alternativa al carcere. L'esperienza europea", in Rassegna penitenziaria e criminologica, n. 2, 2013, pp. 79124.

15 Buls D., Freeman R., DHSRU University of Dundee, Qualitative interviews: Expert opinion on alternatives to custody in Scotland, 2015, consultabile al sito:

http://www.reducingprison.eu/downloads/files/inte rviews-report-Scotland.pdf

${ }^{16}$ Ferrarella L., "Finiti i braccialetti elettronici, scatta il numero chiuso", Il Corriere della sera, 3 luglio 2014. Disponibile alla pagina: http://www.corriere.it/cronache/14_luglio_03/finiti -braccialetti-elettronici-scatta-numero-chiusodb2b7678-0272-11e4-af6d-a9a93b39a7aa.shtml nel corso dell'intervista, ha posto l'accento su come l'utilizzo di questi dispositivi sia troppo condizionato dalla disponibilità degli stessi: sul piano culturale, il giudice deve decidere sulla base degli atti se una persona è meritevole o no di uscire dal carcere e condizionare la libertà, alla disponibilità dei dispositivi elettronici è considerata in maniera par cosa gravissima poiché il giudizio sulla pericolosità e sulla libertà di una persona non può dipendere da un fatto meccanico e organizzativo.

\section{Il dibattito sulle alternative al carcere.}

Nei paesi coinvolti dal progetto le alternative alla detenzione, sia in fase cautelare che definitiva, non sono esplicitamente indirizzate a un target specifico: l'accesso è determinato in funzione prevalente dell'entità della pena inflitta e dunque consentito per i reati di minor allarme sociale.

L'eccessivo affollamento degli istituti di pena, la mancanza di efficacia della reclusione nella riduzione della recidiva e l'abbattimento dei costi sono, in generale, le principali motivazioni che sostengono l'utilizzo delle misure alternative in tutti i paesi partecipanti al progetto. In Italia e Scozia, altri argomenti di discussione riguardano la finalità riabilitativa della pena, in relazione alla quale la detenzione è sicuramente meno efficace, e la necessità che la pena abbia la finalità di riparare il danno causato dal reato.

Il rapporto sul quadro politico delle alternative alla detenzione in ambito europeo ha rilevato come, nonostante le alternative al carcere siano promosse in tutti gli Stati membri, esistano alcune importanti differenze tra i paesi europei 
in materia di obiettivi e finalità delle stesse $\mathrm{e}^{17}$. Secondo McNeill queste differenze sono sorte poiché, in molte giurisdizioni, le sanzioni alternative sono considerate misure imposte al posto della reclusione (considerata la "vera punizione") o forme di pena sospesa, e quindi non sono percepite come una condanna vera e propria $^{18}$.

Nei paesi dell'est europeo, ad esempio, il consolidamento di misure non custodiali è più attuale. In Romania, il nuovo codice penale entrato in vigore nel febbraio 2014 per sostituire il precedente di matrice socialista, ha apportato modifiche sostanziali rispetto alle alternative alla detenzione applicabili durante il processo, stabilendo, tra l'altro, condizioni più restrittive per disporre la sospensione condizionale della pena con la messa alla prova.

In Bulgaria, invece, lo sviluppo di misure non carcerarie non è stato la conseguenza di dibattiti interni, ma è derivato dalla necessità di sincronizzare la legislazione del paese con quella comunitaria, nell'ottica del processo di adesione all'Unione Europea ${ }^{19}$. In Lettonia la democrazia è relativamente recente e le politiche penali risalgono a metà degli anni 1990: per lungo tempo i dibattiti sulle alternative alla detenzione sono stati praticamente inesistenti, tuttavia il

17 Snacken S., McNeill F., Chapter 2: Scientific Reccommendations, in. Flore S., Bosly A., Honhon \& J. Maggio, Probation Measures and Alternative Sanctions in the European Union, pp. 561-571, Intersentia, Cambridge, 2012, citato da De Vos H., Gilbert E., Aertsen I, op. cit.

${ }_{18}$ McNeill F., "Community Sanctions and European Penology", in Daems T., Snacken S., Van Zyl Smit D., European penology, Hart Publishing, Oxford, 2013, citato da De Vos H., Gilbert E., Aertsen I., op.cit.

19 Momchilov A., Rusinov D. (Crime Prevention Fund-IGA), National report Bulgaria, 2014, consultabile al sito: http://www.reducingprison.eu/downloads/files/inte rview_report_Bulgaria.pdf sovraffollamento e gli altri tassi di recidiva hanno portato allo sviluppo di una nuova legislazione che prevede alternative alla pena detentiva e specifici programmi di risocializzazione nelle carceri. Nel sistema lettone, tuttavia, la privazione della libertà non è considerata la "pena principale" per la quale possono esserci delle alternative, ma esistono pene specifiche che non prevedono l'isolamento della persona dalla società e diverse modalità di applicazione del rilascio prima dell'aver finito di scontare una condanna in carcere ${ }^{20}$.

La società civile attraverso le ONG è molto attiva nel settore delle alternative alla detenzione in tutti $i$ paesi coinvolti nel progetto, influenzando il sistema politico, proponendo prassi operative, facendo ricerca sociale, gestendo servizi connessi all'alternativa al carcere. In Italia, in particolare, sono numerose le associazioni del non profit e della cooperazione sociale, tra cui l'Associazione Comunità Papa Giovanni XXXIII, che collaborano attivamente con lo Stato e quindi con gli Uffici di Esecuzione Penale Esterna del Ministero della Giustizia - Dipartimento dell'Amministrazione Penitenziaria, nel favorire il recupero e reinserimento sociale dei soggetti ammessi a beneficiare di una misura alternativa alla detenzione. Secondo una recente indagine condotta da Fondazione Volontariato e Partecipazione (FVP) e del Centro Nazionale per il Volontariato (CNV), ${ }^{21}$ sono quasi 3000 le

\footnotetext{
20 Kronberga I. (Centre for public policy PROVIDUS), National report on Latvia- alternatives to imprisonment, 2014, consulatabile al sito: http://www.reducingprison.eu/downloads/files/Nat ional $\% 20$ Report $\% 20$ on $\% 20$ Latvia.pdf

${ }^{21}$ Centro Nazionale per il volontariato, Fondazione Volontariato e Partecipazione, La certezza del recupero. I costi del carcere e $i$ benefici delle misure alternative: report di
} 
Associazioni che operano anche sporadicamente e su diversi livelli in ambito penitenziario o comunque in contatto diretto o indiretto con il mondo carcerario anche solo per attività di sensibilizzazione sul territorio.

In Bulgaria il ruolo delle ONG è stato determinante nello sviluppo di progetti volti all'introduzione, dapprima sperimentale, di misure alternative nel $\mathrm{Paese}^{22}$. Se il volontariato in carcere è un'esperienza che ha una storia ormai consolidata, il volontariato nei contesti di probation è sicuramente meno diffuso, nonostante lodevoli eccezioni come, ad esempio, la Romania, dove, ogni anno, sono coinvolti tra i 300 e i 500 volontari nei 42 servizi territoriali di probation del Paese ${ }^{23}$.

Per i detenuti tossicodipendenti, in tutti i Paesi del partenariato sono previste alternative volte a ritardare, evitare, sostituire o integrare la pena detentiva (ad es. lavoro di pubblica utilità, sospensione della patente, trattamento della tossicodipendenza) sulla base del presupposto che le loro esigenze possano essere affrontate meglio con interventi di questo tipo, come raccomandato da tutte le convenzioni e linee guida internazionali. In alcuni paesi queste misure sono poco utilizzate a causa di vincoli giuridici o dello diffidenza nei confronti della loro efficacia.

Il disagio psichico è un elemento di grande rilievo dell'universo penitenziario dei Paesi

sintesi, dicembre 2014, consultabile al sito: http://www.centrovolontariato.net/wpcontent/uploads/2014/12/carcere-report1.pdf

${ }^{22}$ Momchilov A., Rusinov D., op. cit.

23 Asociatia "Generatie Tanara" (Unga-Liv), Summary of the in-depht interviews to country experts, Romania, 2014, consultabile al sito: http://www.reducingprison.eu/downloads/files/inte rviews_report_Romania.pdf coinvolti dal progetto, tuttavia, il dibattito sulla condizione dei detenuti con problemi psichiatrici è molto limitato. La Lettonia e la Bulgaria hanno rilevato la necessità di formazione specifica del personale e l'introduzione di interventi specialistici per detenuti psichiatrici.

\section{Punti di forza e di debolezza.}

L'analisi e il confronto del sistema di giustizia penale e del quadro giuridico sulle misure alternative alla detenzione in uso nei paesi partner ha permesso di individuare alcuni punti di forza e di debolezza che sono comuni a tutti i paesi coinvolti e che dovrebbero essere presi in considerazione da politici e legislatori per un efficace ed efficiente riforma del sistema giudiziario.

Il rapporto costi-benefici è stato analizzato in più occasioni: in Bulgaria, ad esempio, il mantenimento di una persona in carcere è circa 7 volte più costoso dell'applicazione di una sanzione di comunità. Nello stesso Paese, il numero di ore di lavori di pubblica utilità svolte da persone in libertà vigilata è stato più di 770.000, che corrisponde a circa 1 milione di euro per la comunità e al 12-13\% del bilancio del servizio di probation. In Italia, nonostante il costo medio giornaliero a detenuto sia tra $i$ più alti d'Europa, non sono previsti finanziamenti per opere educative, di recupero e di incremento della sicurezza pubblica alternative al carcere. La Comunità Papa Giovanni XXIII da anni sperimenta il progetto CEC "Comunità Educante con i Carcerati" un percorso educativo per detenuti che beneficiano di una misura alternativa al carcere che concepisce la pena 
come occasione riparativa, rieducativa e non vendicativa, proprio come stabilito dalla Costituzione Italiana. Se venisse riconosciuta una retta di 40 euro al giorno a testa dallo Stato, per 10.000 detenuti in un solo anno sarebbe possibile avere un significativo risparmio della spesa pubblica destinata ai detenuti, oltre ad un'importante ricaduta sociale.

Se è vero, poi, che attraverso la pena detentiva la società intende difendere se stessa da chi attenta alla sua stabilità, occorre verificare se il carcere renda effettivamente meno pericolosi i suoi detenuti. Diverse, infatti, sono le voci critiche secondo le quali la carcerazione sancisca la definitiva espulsione del recluso dal tessuto sociale, aggravando l'orientamento deviante e finendo per restituire alla società un individuo peggiore di quello che è entrato ${ }^{24}$.

E' opinione condivisa da tutti i partners che $\mathrm{i}$ vantaggi nell'utilizzo di misure alternative siano rappresentati dal maggior rispetto dei diritti umani e dall'effettiva finalità riabilitativa. Il carattere costruttivo, e non semplicemente limitativo, delle pene alternative riduce la possibilità che il detenuto commetta ulteriori reati, con un aumento esponenziale della sicurezza sociale: un uomo recuperato non è più pericoloso e quindi è evidente il beneficio, sociale ma anche economico, dell'intera collettività.

I limiti delle misure non carcerarie sono riscontrati dagli esperti intervistati nelle eccessive preclusioni legislative alla loro concessione, nella percezione tendenzialmente negativa della società, che non riesce a superare l'equazione pena-carcere, e nella limitata capacità delle istituzioni nel monitorare l'implementazione di queste misure.

Il carcere, in quanto istituzione totale, è ancora ritenuto dal sentire comune come più sicuro e funzionale non solo per chi delinque, ma anche per abbassare i tassi di criminalità. E', inoltre, opinione comune che nella maggior parte dei paesi scarseggino le risorse istituzionali, di personale ed economiche per alimentare il circuito delle sanzioni di comunità. Come rilevato dal report europeo condotto dall'Istituto di Criminologia di Leuven, questo squilibrio dimostra come le Istituzioni non considerino le alternative al carcere efficaci o idonee al pari delle pene detentive. Lo scarso investimento nel settore, poi, implica anche una carenza di personale qualificato, che può portare a conseguenze negative per la supervisione del reo e quindi per il successo della misura ${ }^{25}$. E' invece necessario garantire una formazione adeguata a chi opera in questo settore, e fornire al personale impegnato gli strumenti e le conoscenze necessarie per instaurare e gestire una relazione efficace con le persone condannate.

\section{Il ruolo della vittima.}

Negli ultimi vent'anni in ambito europeo si è diffusa una crescente attenzione per le vittime di reato e per il loro potenziale ruolo nei procedimenti penali e numerosi atti normativi hanno cercato di valorizzarne il ruolo e rafforzarne la tutela, prescrivendo agli Stati l'ampliamento delle sue facoltà processuali, l'adozione di strumenti idonei a prevenire nuove vittimizzazioni e lo sviluppo di meccanismi volti

${ }^{24}$ Campana D., Condannati a delinquere? Il carcere e la recidiva, Franco Angeli, Milano, 2009.

${ }^{25}$ De Vos H., Gilbert E., Aertsen I, op.cit. 
a garantire l'ottenimento di un risarcimento per i danni subiti.

Già nel 1985, il Consiglio d'Europa ha pubblicato una raccomandazione relativa alla posizione della vittima nell'ambito del diritto e della procedura penale $(\mathrm{R}(85) 11)$ con l'obiettivo di suggerire agli Stati membri l'adozione di una serie di misure, volte a tutelare le vittime in tutte le fasi del procedimento, evitando cosi il verificarsi di episodi di vittimizzazione secondaria. La Direttiva 2012/29/Ue stabilendo norme minime "in materia di diritti, assistenza e protezione delle vittime di reato", ha sostituito la precedente e meno articolata Decisione Quadro 2001/220/GAI UE “sulla posižone della vittima nel procedimento penale". La Direttiva riconosce alla vittima numerosi diritti in tutto l'arco processuale, inclusa l'esecuzione penitenziaria: dal diritto ad ottenere dettagliate, comprensibili informazioni sul proprio caso al diritto di accesso ai servizi di assistenza, dai diritti di partecipazione al procedimento penale al diritto ad una variegata protezione. Tra i molti diritti, la Direttiva riconosce alla vittima anche "il diritto a garanzie nel contesto dei servizi di giustizia riparativa".

La giustizia riparativa ha tra $\mathrm{i}$ suoi obiettivi principali la presa in carico dei bisogni delle vittime di reato, che, storicamente, hanno sempre occupato una posizione marginale all'interno del processo penale.

Il valore della giustizia riparativa, e soprattutto della mediazione è sempre più riconosciuto e tali modelli esistono in alcuni Paesi, anche se la fiducia nel loro successo non è costante. Il rapporto sul quadro politico delle alternative alla detenzione in ambito europeo ha mostrato come numerosi studi sostengano che la mediazione penale e, più in generale, le pratiche di giustizia riparativa siano particolarmente efficaci per i reati gravi, e che l'effetto dell'uso di tali pratiche sulla recidiva sia maggiore se applicato a reati gravi.26

Tutti i Paesi riconoscono il ruolo della vittima nel procedimento penale, anche se Bulgaria e Lettonia raramente incoraggiano la sua partecipazione attiva al processo. La Scozia è probabilmente il paese più all'avanguardia in questo settore in quanto ha recentemente adottato una normativa "Victims and Witnesses (Scotland) Act 2014" che riconosce la vittima del reato non solo come spettatrice passiva o destinataria di servizi del sistema giudiziario, ma come persona che ha un legittimo interesse nel funzionamento del sistema e che merita un posto di rilievo all'interno del processo.

In Italia e in Germania, invece, la vittima ha un ruolo più marginale e non è coinvolta nel procedimento di applicazione della misura alternativa alla detenzione. Un esperto italiano intervistato nell'ambito del progetto ha messo in luce come nel Paese manchi una "cultura della vittima": questo, secondo lui, perché l'amministrazione penitenziaria, valutando l'attività del giudice dal punto di vista della produttività, in un'ottica prevalentemente quantitativa, non induce quest'ultimo a tener conto della vittima, salvo $i$ casi in cui questa si presenti come parte civile e chieda il risarcimento del danno. Lo stesso esperto ha manifestato la necessità di un cambiamento culturale per ribaltare la prospettiva e non rimanere indifferenti nei confronti di chi ha 
subito le conseguenze di un reato. ${ }^{27}$ In Francia e in Germania, infatti, sono stati soprattutto i movimenti per la tutela delle vittime a sollecitare l'attenzione sul tema della giustizia riparativa, e in Lettonia gli esperti intervistati per il progetto hanno evidenziato l'importanza e la necessità di tali pratiche per riconoscere alla vittima la sofferenza subita, la sua dignità e i suoi bisogni. ${ }^{28}$ La mediazione penale è lo strumento principale per l'applicazione concreta del modello riparativo: esperienze di mediazione vittima-reo sono presenti nei paesi coinvolti dal progetto, ma il loro sviluppo non è omogeneo. La mediazione si pone come un sistema complementare al procedimento penale tradizionale o come un'alternativa ad esso: il codice penale tedesco, ad esempio, prevede la mediazione vittima-autore del reato come alternativa alla detenzione, come condizione per la libertà vigilata o per l'uscita anticipata dal carcere $^{29}$.

Un'ulteriore esigenza, per la reale tutela delle vittime, riguarda il coinvolgimento della comunità secondo una metodologia di rete tra le istituzioni e le associazioni del territorio, che

\footnotetext{
26 Sherman L. W., Strang H., Restorative justice: the evidence, The Smith Institute, London, 2007.

27 Stefani G., Associazione Comunità Papa Giovanni XXIII, Report sulle interviste effettuate a cinque esperti italiani nel settore delle alternative alla detenzione, 2015, consultabile al sito: http://www.reducingprison.eu/downloads/files/RE PORT_ITALIANO \%20.pdf

${ }^{28}$ Sile S., Centre for public policy PROVIDUS; Alternatives to imprisonment in Latvia, 2015, consultabile al http://www.reducingprison.eu/downloads/files/inte rview_report_Latvia.pdf

${ }^{29}$ Bähr A., Hartmann A., Steengrafe F., University of Applied Science for Public Administration of Bremen, National Report - Germany, 2014, consultabile al sito: http:/ /www.reducingprison.eu/downloads/files/Nat ional $\% 20$ Report $\% 20-\% 20$ Germany.pdf
}

possono porsi come promotori di interventi di riconciliazione, di sostegno alle vittime, di riabilitazione degli autori di reato, realizzando fattivamente politiche di inclusione sociale. Il coinvolgimento della comunità, inoltre, può indubbiamente portare ad una maggiore consapevolezza dell'opinione sulle conseguenze dannose del reato e, parallelamente, incoraggiare attività di prevenzione del crimine.

\section{L'individuazione di buone pratiche di} alternativa alla detenzione.

Negli ultimi anni la Commissione europea ha incentivato le iniziative per promuovere l'individuazione di buone pratiche, utili da condividere e sostenere affinché tali esperienze possano alimentarne di nuove in altri contesti, o rappresentino un riferimento efficace per prendere spunti e informazioni utili ad avviare sviluppi innovativi alle proprie iniziative, o per essere adattate al proprio contesto locale e alle proprie esigenze. L'analisi della letteratura nazionale e le interviste agli esperti condotte nei paesi coinvolti hanno permesso di compiere un approfondimento sulle attuali alternative al carcere e di identificare alcuni criteri per la selezione delle buone pratiche nel settore delle misure non carcerarie.

I criteri che sono stati elaborati da Synergia e condivisi dal partenariato sono i seguenti: ${ }^{30}$

- E' necessario che ogni pena sia adatta alle caratteristiche dell'accusato e/o del condannato. La scelta della misura alternativa da applicare dovrebbe avvenire

\footnotetext{
30 Synergia, In-depht analysis of ggod practices by country, 2015, consultabile al sito: http://www.reducingprison.eu/downloads/files/Cas e_studies_reports.pdf
} 
sulla base dei suoi potenziali effetti sulla persona condannata/accusata e, quindi, dipende da ogni singolo caso. In quest'ottica, per identificare la pena più appropriata per ogni individuo è necessario conoscere la persona, la sua personalità, (i suoi bisogni, i suoi rischi, le sue esperienze, la sua capacità di capire cosa sia socialmente accettabile) e il percorso che vuole intraprendere.

- Le alternative al carcere dovrebbero essere personalizzabili a seconda della pericolosità e dei bisogni del condannato e dovrebbero avere un impatto sul suo modo di pensare, sui suoi valori e sulla sua capacità di capire cosa sia socialmente accettabile. In altre parole, dovrebbero avere una finalità riabilitativa.

- E' necessario che le alternative al carcere siano caratterizzate da un approccio flessibile che possa venire incontro ai bisogni dell'individuo e che permetta di monitorare, rivedere e, se necessario, modificare la pena a seconda dei progressi dell'individuo

- E' importante che tra l'autore di reato e il suo supervisore si instauri una relazione di fiducia: la loro relazione dovrebbe essere credibile agli occhi del condannato e basata sull'ascolto attivo, sull'empatia e sulla comunicazione interpersonale.

Sulla base di questi criteri ogni gruppo nazionale ha identificato tre buone pratiche nel proprio paese, successivamente approfondite con dei casi studio $^{31}$. Le pratiche selezionate dai partners sono state classificate da Synergia in quattro gruppi:

1. Misure in cui il focus ̀̀ la riabilitazione del condannato attraverso la predisposizione di percorsi educativi e psicologici

Rientrano in questa categoria la pratica italiana CEC "Comunità Educante con $i$ Carcerati" dell'Associazione Comunità Papa Giovanni XXIII, che propone ai detenuti un percorso educativo in una dimensione di casa e di famiglia, la bulgara C.S.R.I.O. "Center for Social Rehabilitation and Integration of Offenders", un centro per autori di reato gestito da IGA Foundation che offre diversi servizi, le scozzesi "Caledonian system" e "Moving Forward Making Change" che offrono programmi residenziali indirizzati, rispettivamente, agli autori di violenza domestica e di crimini sessuali.

Gli aspetti positivi di queste misure sono stati identificati nella valutazione delle motivazioni dell'autore di reato, nella presenza di percorsi strutturati personalizzati e supervisionati da un operatore, e nel miglioramento del capitale sociale dell'individuo.

\section{Misure che si concentrano sul recupero dell' individuo attraverso percorsi lavorativi.}

L'inserimento lavorativo, attraverso percorsi specifici di formazione e di lavoro, è uno strumento basilare per favorire l'integrazione del detenuto all'interno della società in modo dignitoso e gratificante. Le pratiche che fanno parte di questa categoria sono numerose. La tedesca HSI "Haftvermeidung durch soziale Integration", ad esempio, attraverso una serie di organizzazioni che lavorano in rete, offre diversi 
servizi al fine di favorire la soluzione dei problemi sociali di detenuti ed ex-detenuti. Il progetto AC.E.RO "Accoglienza e Lavoro", promosso dalla Regione Emilia-Romagna e dal Provveditorato dell'Amministrazione

Penitenziaria della stessa Regione attraverso attività di accoglienza in strutture riabilitative collettive individuate sul territorio dell'EmiliaRomagna e percorsi di inclusione lavorativa intende fornire competenze e abilità per l'acquisizione e il consolidamento del livello di autonomia di persone dimesse dal carcere per l'ammissione ad una misura alternativa, al fine di contenere il rischio di recidiva. In Bulgaria la normativa prevede la possibilità di lavorare $\mathrm{O}$ studiare al fine di ridurre la durata della pena detentiva: questo è un importante incentivo per motivare i detenuti all'acquisizione di competenze formative e professionali. Il francese "Accompagnement renforcê", inizialmente sperimentato nella municipalità di Cambrai e successivamente esportato a Beauvais e SaintQuentin, prevede un aggiunta al servizio di probation attraverso attività di sostegno come, ad esempio, il supporto nella stesura del curriculum vitae, nel disbrigo di pratiche burocratiche, visite a domicilio ecc. Le misure che propongono percorsi di questo tipo hanno in comune $\mathrm{i}$ seguenti punti di forza: l'importanza di acquisire competenze professionali per raggiungere la piena autonomia, la metodologia di lavoro in rete che permette all'individuo di trovare facilmente un'occupazione, la consapevolezza che il lavoro non può essere l'unico obiettivo ma che deve, comunque, essere accompagnato da un percorso di reintegrazione sociale.

\footnotetext{
31 Ibidem.
}

\section{Azioni di giustizia riparativa e di servizio alla} comunità.

La presa in carico dei bisogni delle vittime di reato sono gli obiettivi fondamentali delle pratiche che prevedono azioni riparative. Il progetto "RiparAqioni”, implementato a Mantova dall'Associazione Libra Onlus si pone l'obiettivo di diffondere la conoscenza degli strumenti di restorative justice e di realizzare percorsi di responsabilizzazione per detenuti in misura alternativa che possano sfociare in concrete azioni riparatorie per la vittima o per la società in generale. Lo svolgimento di un'attività lavorativa a beneficio della collettività può costituire una forma di riparazione attuata verso la società, considerata parte offesa del reato: azioni di questo tipo sono stati individuate come buone pratiche in Bulgaria, in Lettonia e in Romania. In Germania, il servizio alla comunità è parte fondamentale della buona pratica tedesca implementata dall'Associazione "Bruecke Bremen", che cerca di evitare, quando possibile, l'esperienza carceraria proponendo il lavoro di pubblica utilità. Le pratiche che rientrano in questa categoria hanno un'indubbia valenza sia per il reo, promuovendo la sua reintegrazione sociale, sia per la vittima (diretta o indiretta), sono inoltre flessibili e facilmente monitorabili e non allontanano l'autore di reato dal suo ambiente sociale e familiare.

\section{Misure giuridiche alternative alla detenzione adattate alle esigenze e ai rischi del reo.}

Le misure giuridiche considerate "buone pratiche" che rientrano in questa categoria sono l'Articolo 723-15 della procedura francese, che mira ad evitare l'esperienza carceraria per i condannati a pene detentive medio-brevi e il JAP 
(Juges de l'application des peines) il giudice dell'applicazione delle pene, che costituisce la giurisdizione di primo grado in Francia ed è incaricato di fissare le principali modalità di esecuzione delle pene privative della libertà, disponendo e controllando le condizioni della loro applicazione. In Romania, la procrastinazione della pena, introdotta nel 2014, offre alla corte la possibilità di prendere in considerazione elementi oggettivi e soggettivi sull'autore del reato al momento della scelta della sanzione. In Lettonia il rilascio condizionale permette al condannato l'uscita anticipata dal carcere qualora si ritenga che quest'ultimo sia in grado di adattarsi alla società senza commettere ulteriori reati. Queste buone pratiche evidenziano l'importanza della personalizzazione della pena a seconda delle circostanze del reato e della personalità del trasgressore, tuttavia per un'efficace applicazione sono necessarie risorse che permettano di garantire un buon livello di vigilanza e controllo.

\section{Osservazioni conclusive.}

L'attività di ricerca sinora condotta ha permesso di ottenere un quadro generale su come le alternative al carcere possano contribuire ad affrontare in maniera efficace $i$ problemi che attanagliano sistema carcerario europeo: il sovraffollamento, il dispendio economico, la recidività e la tutela delle vittime.

La complessità del fenomeno e i fattori che lo influenzano, richiedono che ogni paese elabori una propria strategia, tenendo in primo luogo presente che le misure non carcerarie devono essere considerate una vera e propria sostituzione alla pena detentiva: non un premio, ma un percorso serio e severo volto alla riabilitazione del condannato. In questa prospettiva è necessario il monitoraggio continuo della corretta applicazione della misura, un sostegno finanziario sufficiente e personale formato al compito: naturalmente, questo approccio deve essere collocato in un più ampio contesto di politiche sociali che promuova il reinserimento dell'autore di reato, e che sia attento ai bisogni e ai diritti della vittima.

La Comunita Papa Giovanni XXIII, da anni tenta di rispondere all'emergenza carceri sviluppando su tutto il territorio nazionale comunità educative volte all'accoglienza di detenuti, con l'obiettivo di passare dalla certezza della pena alla certezza del recupero, concependo quindi la pena come strumento di integrazione e non di ulteriore esclusione del soggetto deviante dal contesto sociale. La metodologia utilizzata intende valorizzare gli elementi positivi presenti nella persona, nella convinzione che da lì possa partire il riscatto e il pieno reinserimento nella società.

A livello politico sono necessarie, tuttavia, scelte politiche coraggiose in grado di colmare il vuoto che separa la condizione carceraria dalla finalità rieducativa della pena e dall'effettiva tutela dei diritti e della dignità della persona.

Le attività del progetto "Reducing Prison Population: advanced tools of justice in Europe" proseguiranno attraverso la definizione delle Linee Guida per l'implementazione delle alternative alla detenzione e di un Manuale Operativo per la formazione del personale impegnato, a vario titolo, nell'implementazione delle misure non carcerarie, documenti che saranno tradotti in 
tutte le lingue dei partners progettuali e divulgate attraverso il sito internet dedicato.

\section{Bibliografia.}

- $\quad$ Aebi M.F., Chopin J., SPACE II- Council of Europe Annual Penal Statistics: Persons Serving non-custodial sanctions and measures in 2013. Survey 2013, Consiglio d'Europa, Strasburgo, 2015.

- Aebi M.F., Delgrande N., SPACE I Council of Europe Annual Penal Statistics: Prisons populations, Survey 2013, Consiglio d'Europa, Strasburgo, 2015.

- Asociatia "Generatie Tanara" (Unga-Liv), National report-Romania, 2014. Disponibile al sito:

http://www.reducingprison.eu/downloads/ files/National $\% 20$ report $\% 20$ Romania.pdf

- Asociatia "Generatie Tanara" (Unga-Liv), Summary of the in-depht interviews to country experts, Romania, 2015. Disponibile al sito: http://www.reducingprison.eu/downloads/ files/interviews_report_Romania.pdf

- Associazione Antigone, Oltre i tre metri quadri. XI Rapporto sulle condizioni di detenzione, EGA, Torino, 2015.

- Bähr A., Hartmann A., Ede K., University of Applied Science for Public Administration of Bremen, National Report Germany, In-depth interviews with k-speakers, 2015. Disponibile al sito: http://www.reducingprison.eu/downloads / files/Interview_report_Germany.pdf

- Bähr A., Hartmann A., Steengrafe F., University of Applied Science for Public Administration of Bremen, National Report Germany, 2014. Disponibile al sito: http://www.reducingprison.eu/downloads/ files $/$ National $\% 20$ Report $\% 20$ -

\%20Germany.pdf

- Balloni A. (a cura di), Vittima, crimine, difesa sociale, Clueb, Bologna, 1990.

- Balloni A., Mosconi G., Prina F. (a cura di), Cultura giuridica e attori della giustizia penale, FrancoAngeli, Milano, 2004.

- Balloni A., Bisi R., Sette R., Principi di Criminologia - Le Teorie, Cedam, Padova, 2015.

- Balloni A., Bisi R., Sette R., Principi di Criminologia - Criminalità, Controllo, Sicurezza, Cedam, Padova, 2015.

- Beyens K., Snacken S. Eliaerts C., Barstende muren, overbevolkte gevangenissen: omvang, oorzaken en mogelijke oplossingen, Kluwer, Bruxelles, 1993.

- Bisi R., Operatori penitenziari a confronto, Clueb, Bologna, 1990.

- Bisi R., Faccioli P. (a cura di), Con gli occhi della vittima. Un approccio interdisciplinare alla vittimologia, FrancoAngeli, Milano, 1996.

- Buls D., Freeman R., DHSRU University of Dundee, National reports on alternatives to imprisonment in Scotland, 2014. Disponibile al sito:

http://www.reducingprison.eu/downloads / files $/$ National $\% 20$ report $\% 20$ -

UK_Scotland2.pdf

- Buls D., Freeman R., DHSRU University of Dundee, Qualitative interviews: Expert opinion on alternatives to custody in Scotland, 2015. Disponibile al sito: http://www.reducingprison.eu/downloads / files/interviews-report-Scotland.pdf

- Campana D., Condannati a delinquere? Il carcere e la recidiva, Franco Angeli, Milano, 2009.

- Centro Interdisciplinare di Ricerca sulla Vittimologia e sulla Sicurezza, Università di Bologna (CIRVIS-SDE), National Report on Italy, 2014. Disponibile al sito: http://www.reducingprison.eu/downloads / files/National $\% 20$ Report $\% 20$ Italy.pdf

- Centro Nazionale per il volontariato, Fondazione Volontariato e Partecipazione, La certezza del recupero. I costi del carcere e $i$ benefici delle misure alternative: report di sintesi, 2014. Disponibile al sito: http://www.centrovolontariato.net/wpcontent/uploads/2014/12/carcerereport.pdf

- Commissione Europea, Libro Verde. Rafforzare a fiducia reciproca nello spazio giudiziario europeo — Libro verde sull'applicazione della normativa dell'UE sulla giustizia penale nel settore della detenzione, 2011. Disponibile al sito:

http://www.europarl.europa.eu/meetdocs/ 2009 2014/documents/com/com com (2011)0327_/com_com(2011)0327_it.pdf

- De Vos H., Gilbert E., Aertsen I., Reducing prison pupolation overview of the legal and policy framework on alternatives to imprisonment at the European level, 2014. Disponibile al sito: http://www.reducingprison.eu/downloads/ files/ReducingprisonpopulationEuropeanfra mework FIN 101014.pdf

- Ferrarella L., "Finiti i braccialetti elettronici, scatta il numero chiuso", Il Corriere della sera, 3 luglio 2014. Disponibile alla pagina: http://www.corriere.it/cronache/14_luglio_ 
03/finiti-braccialetti-elettronici-scattanumero-chiuso-db2b7678-0272-11e4-af6da9a93b39a7aa.shtml

- Gonnella P., Detenuti stranieri in Italia. Norme, numeri e diritti, Editoriale scientifica, Napoli, 2015.

- Herzog Evans M. under the auspices of the International Society for Criminology, Alternatives to incarceration in France, 2015. Disponibile al sito: http://www.reducingprison.eu/downloads/ files/224_Finale_Translation_of_Evans Boesel_Report_on_France.pdf

- Herzog Evans M. under the auspices of the International Society for Criminology, Ten Existing Practices on Alternatives to ImprisonmentReport on France, 2015. Disponibile al sito: http://www.reducingprison.eu/downloads/ files/interview_report_France.pdf

- Herzog Evans M., "What's in a name: Penological and institutional connotations of probation officers' labelling in Europe", in EuroVista, n. 2, 2013, pp. 121-133.

- Kronberga I. (Centre for public policy PROVIDUS), National report on Latviaalternatives to imprisonment, 2014.

- Leonardi F., "Le misure alternative alla detenzione tra reinserimento sociale $\mathrm{e}$ abbattimento della recidiva", in Rassegna penitenziaria e criminologica, n. 2, Anno XI Maggio-Agosto 2007, pp. 7-26.

- Leonardi F., "La sorveglianza elettronica come alternativa al carcere. L'esperienza europea", in Rassegna penitenziaria $e$ criminologica, n. 2, 2013, pp. 79-124.

- Martufi A., "Sovraffollamento carcerario e alternative alla detenzione: esperienze europee a confronto. Dialogo tra Italia, Belgio, Francia, Polonia, Romania e Spagna", in Rivista Italiana di Diritto e Procedura Penale, Anno LVIIN, Fasc. 4, 2014.

- McNeill F., "Community Sanctions and European Penology", in Daems T., Snacken S., Van Zyl Smit D., European penology, Hart Publishing, Oxford, 2013.

- Momchilov A., Rusinov D. (Crime Prevention Fund-IGA), National report Bulgaria, 2014. Disponibile al sito: http://www.reducingprison.eu/downloads/ files/interview_report_Bulgaria.pdf

- Momchilov A., Rusinov D. (Crime Prevention Fund-IGA), Qualitative interviews with k-actors, 2015. Disponibile al sito: http://www.reducingprison.eu/downloads/ files/Interviews $\% 20$ report $\% 20$ Bulgaria.pdf
- Osservatorio Europeo Droghe e Tossicodipendenze (OEDT), Relarione europea sulla droga. Tendenze e sviluppi, 2014. Disponibile al sito: http://www.emcdda.europa.eu/attachement s.cfm/att_228272_IT_TDAT14001ITN.pdf

- Sette R., Criminologia e vittimologia. Metodologie e strategie operative, Minerva, Bologna, 2011.

- Sette R., "Le cure materne e il reinserimento sociale della condannata: attualità di un vecchio problema", in Rivista di Criminologia, Vittimologia e Sicurezza, vol. VIII, n. 3, settembre-dicembre 2014, pp. 56-73 (DOI: http://dx.medra.org/10.14664/rcvs/143), disponibile sul sito Internet: www.vittimologia.it/rivista

- Sherman L. W., Strang H., Restorative justice: the evidence, The Smith Institute, London, 2007.

- Sile S., Centre for public policy PROVIDUS; Alternatives to imprisonment in Latvia, 2015. Disponibile al sito: http://www.reducingprison.eu/downloads/ files/interview_report_Latvia.pdf

- Snacken S., McNeill F., Chapter 2: Scientific Reccommendations, in. Flore S., Bosly A., Honhon \& J. Maggio, Probation Measures and Alternative Sanctions in the European Union, pp. 561-571, Intersentia, Cambridge, 2012.

- Stefani G., Associazione Comunità Papa Giovanni XXIII, Report sulle interviste effettuate a cinque esperti italiani nel settore delle alternative alla detenzione, 2015. Disponibile al sito: http://www.reducingprison.eu/downloads/ files/REPORT_ITALIANO \%20.pdf

- Synergia, Comparative concluding remarks of the in -depth interviews to the country-experts, 2015. Disponibile al sito: http://www.reducingprison.eu/downloads/ files/final_research_conclusions 3.pdf

- Synergia, In depht analysis of good practices by country, 2015. Disponibile al sito: http://www.reducingprison.eu/downloads/ files/Case_studies_reports.pdf

- Synergia, Selected good practices for the analysis of case studies by country, 2015. Disponibile al sito:

http://www.reducingprison.eu/downloads/ files/WP2 list $\% 20$ of $\% 20$ good $\% 20$ practice s.pdf 